\title{
New techniques for sentinel node biopsy in breast cancer
}

\author{
Massimo Ferrucci ${ }^{1}$, Gianluca Franceschini ${ }^{1}$, Michael Douek $^{2,3}$ \\ ${ }^{1}$ Multidiciplinary Breast Center, Fondazione Policlinico Universitario “Agostino Gemelli”, Università Cattolica del Sacro Cuore, Rome, Italy; \\ ${ }^{2}$ Division of Cancer Studies, Department of Research Oncology, King's College London, London, UK; 'Guy's \& St. Thomas' Hospitals NHS \\ Foundation Trust, London, UK \\ Contributions: (I) Conception and design: M Ferrucci, M Douek; (II) Administrative support: None; (III) Provision of study materials or patients: M \\ Ferrucci; (IV) Collection and assembly of data: M Ferrucci; (V) Data analysis and interpretation: All authors; (VI) Manuscript writing: All authors; (VII) \\ Final approval of manuscript: All authors. \\ Correspondence to: Dr. Massimo Ferrucci, MD. Multidiciplinary Breast Center, Seventh Floor, “O” area, Fondazione Policlinico Universitario \\ “Agostino Gemelli”, Università Cattolica del Sacro Cuore, Largo Francesco Vito n. 1, 00168 Rome, Italy. Email: massimo_ferrucci@hotmail.com; \\ massimo.ferrucci@unicatt.it.
}

\begin{abstract}
Sentinel lymph node biopsy (SLNB) is still the standard of care for axillary nodal staging in patients with invasive breast cancer (BC) and clinically negative lymph nodes (LNs). It successfully replaced the more invasive and morbid axillary lymph node dissection (ALND). The actual standard for SLNB is the radioisotope (RI) with or without blue dye (BD) technique. Because of several drawbacks reported in worldwide experiences, new techniques have been developed in the last years: indocyanine green (ICG) fluorescence, superparamagnetic iron oxide (SPIO) nanoparticles and contrast-enhanced ultrasound (CEUS) using microbubbles. Whilst each technique has its own advantages/disadvantages they are increasing their efficacy and are candidate to represent a new standard for SLNB in next future. This is a comprehensive review of current limitations of conventional techniques besides the improvements and innovations of new methods which, anyway, need future randomized controlled trials to be fully validated.
\end{abstract}

Keywords: Breast cancer (BC); sentinel node biopsy; indocyanine green (ICG); superparamagnetic iron oxyde; microbubble

Submitted Nov 02, 2017. Accepted for publication Feb 01, 2018.

doi: $10.21037 /$ tcr.2018.02.07

View this article at: http://dx.doi.org/10.21037/tcr.2018.02.07

\section{Introduction}

According to sentinel lymph node (SLN) hypothesis, tumor cells migrate in an orderly manner from a primary tumour, metastasizing to one or a few $\mathrm{LNs}$ before involving others. Therefore, SLN is defined as the first LN that receive lymphatic drainage from the primary tumour, if the tumour has spread. The advent of percutaneous lymphoscintigraphy allowed Robinson et al. identify lymphatic drainage patterns of melanomas and the development of SLNB technique through intraoperative lymphatic mapping (1).

Locoregional spread of breast cancer (BC) occurs mainly through the lymphatic system. SLN status accurately predicts the status of the other LNs and is important to establish staging and prognostic outcomes of BC.
SLNB, as ideal nodal staging method for BC, was introduced by Krag et al. and Giuliano et al. in 1993 and 1994 (2,3). Krag et al. described and developed the gamma probe localization of SLN with RI; Giuliano et al. described the SNB using BD alone as a procedure technically feasible, safe, and highly accurate to stage regional LNs in BC (4). SNB has since become the new standard of care for axillary staging in clinically and radiologically node-negative BC (5). Clinical trials demonstrated that in these kinds of patients a properly performed SLNB is equivalent to axillary lymph node dissection (ALND) for staging of axilla (6-8), identifying patients who need further axillary clearance, while sparing others a potentially morbid ALND (9).

This paper is a comprehensive review of the currently used techniques for SLNB with a specific focus on the new 
innovative ones.

\section{Current standard of care for SLNB-blue dye and/or technetium labelled nanocolloid}

The traditional SLNB techniques proposed by Giuliano et al. (4) and Krag et al. (3) have been developed both as single technique and as dual complementary procedure: the choice is determined by surgeon and institutional preference. Giuliano et al. reported a 93\% SLN identification rate using BD alone, while Krag et al. reported a $82 \%$ SLN identification rate using only RI and gamma probe.

James et al. (10) accurately described best practices for both of these tracers besides to the main problems linked to them.

Three types of BD have been described: isosulfan blue, methylene blue (MB) and patent blue. Each surgeon should look for the method that works best for his or her practice, even though isosulfan and patent blue seem to cause a higher rate of adverse reactions (11). Indeed one of the major problem of $\mathrm{BD}$ is a possible adverse reaction up to an anaphylaxis. In case of isosulfan blue it has been reported in $0.7 \%$ to $1.1 \%$ of cases $(12,13)$. $\mathrm{MB}$ has a reduced risk of anaphylaxis but showed side effects as well $(14,15)$ : skin necrosis and induration with associated pain, up to pulmonary edema and serotonin syndrome in patients who take serotonergic medications (16). To reduce the potential side effects, the BD are routinely diluted with normal saline (practices vary from 1:1 to 1:7 dilution) (17). Patient should be screened for known allergies and routine prophylaxis is not suggested as a standard but addressed to high risk cases. The injection site has been widely discussed: peripherically to the tumor, in correspondence of the palpable edge of the biopsy cavity, in the periareolar site or into the subareolar plexus. Chagpar et al. showed that subareolar and periareolar injection produced higher SLN identification rates than peritumoral injection (18). Even though some authors claim that MB diffuses more rapidly in peripheral tissues, staining a larger portion of the breast with the $\mathrm{BD}$ and, to a certain extent, hampering the procedure $(19,20)$, other authors have reported similar accuracy and SLN detection rates (DRs) with MB and with patent blue (21). MB is also much more readily available in different hospitals and less expensive than the others (the cost of MB may represent as little as $3 \%$ of the cost of patent blue or isosulfan blue) (22). Another advantage of $\mathrm{MB}$ is the possibility to be used during pregnancy (23). In a recent prospective randomized trial $\mathrm{MB}$ performed as well as patent blue in identifying SLN in BC (24). MB has so become a standard widely used in USA. Krikanova $e t$ al. reported a 94.6\% SLN identification rate using BD alone making this technique very attractive in high volume units without nuclear medicine facilities or where these facilities are available but when added cost and logistical issues regarding transport of tracer are problematic (25).

The most used RI tracer is the Technetium $99 \mathrm{~m}\left({ }^{99 \mathrm{~m}} \mathrm{Tc}\right)$, ${ }^{99 \mathrm{~m}} \mathrm{Tc}$-sulfur colloid in USA and ${ }^{99 \mathrm{~m}} \mathrm{Tc}$-nanocolloid human serum albumin in Europe. It can be injected before surgery around the tumor, intradermally or into the subareolar plexus. Intradermal injection of radiocolloid appears to be superior to subdermal injection (26-28). Recently Berrocal et al. described the intraoperative injection of ${ }^{99 \mathrm{~m}} \mathrm{Tc}$ in a large series of patients: they reported this method as convenient, effective, safe, and comfortable for the patient with a SLN DR that was essentially $100 \%$ (29). A handheld scintillation counter (gamma probe) is used to guide the surgeon to the labeled LNs. LNs are removed following the " $10 \%$ rule" (all LNs with counts $>10 \%$ of ex vivo count of the most radioactive node should be removed) (30).

The use of RI creates logistical challenges for hospitals, including the handling and disposal of isotopes, training of staff and legislative requirements. The $6 \mathrm{~h}$ half-life of the isotope restricts scheduling of surgery because the injection is done by the nuclear medicine department. Additionally, patients might express reluctance to being exposed to radiation and not all of them (mainly the frail patients) have the possibility to reach an hospital with access to RIs (31). These factors have limited the uptake of SLNB worldwide for hospitals without access to RIs (29). Growing interest is arising in development of new and possibly more effective radio-tracers. New ${ }^{99 \mathrm{~m}} \mathrm{Tc}$-tilmanocept $\left.{ }^{99 \mathrm{~m}} \mathrm{Tc}-\mathrm{TM}\right)$ showed similar SLN uptake to ${ }^{99 \mathrm{~m}} \mathrm{Tc}-$ sulfur colloid but with a rapid injection site clearance, high SLN extraction, low distal node accumulation and no significant pain associated with injection (32). In comparison to ${ }^{99 \mathrm{~m}} \mathrm{Tc}$-nanocolloid human serum albumin used in Europe showed higher localization rate and degree of localization. ${ }^{99 \mathrm{~m}}$ Tc-TM has no contraindications, and no serious adverse or hypersensitivity reactions were reported (33). Another interesting radiotracer has been developed by $\mathrm{Li}$ et al.: ${ }^{99 \mathrm{~m}} \mathrm{Tc}$-rituximab. It contains ${ }^{99 \mathrm{~m}} \mathrm{Tc}-$ labeled monoclonal antibody targeting CD20 abundantly expressed on the surface of B cells in LNs. The advantage is its uniform molecular weight and molecular size so it will not escape easily from SLNs to the second-echelon LNs achieving a clear SLN imaging in patients and high success rate of lymphoscintigraphy. The sensitivity, specificity, and accuracy of SLNB was $97.40 \%$, 
$100 \%$ and $98 \%$, respectively; the $\mathrm{FN}$ rate was $2.60 \%$, which is better than that of other studies (FN rate ranging from 6\% to $10 \%$ ); moreover, its feasibility, safety, and effectiveness have been confirmed by clinical SLNB application with large samples (34). Recently İlem-Özdemir et al. prepared a new RI for SLN mapping which chemically combined a RI and a BD, the ${ }^{99 m} \mathrm{Tc}$-isosulfan blue, to be injected as a single dose. They examined its effectiveness in mapping LNs in rats with promising results (35).

Although excellent results are reported in singleinstitution series using either radioactive colloid or BD $(4,36,37)$ combined use of both tracers appears to be complementary, minimizing the $\mathrm{FN}$ rate in most $(17,18,38)$ but not all, studies (39). Motomura et al. demonstrated that the combination of $\mathrm{BD}$ and RI is superior to dye alone for SLNB in terms of identification rate $(95 \%$ vs. $84 \%)$ and sensitivity (100\% for the combination) (40).

Therefore, the actual gold standard for SLNB is the dual tracer technique which assures higher SLN identification rates and lower FN rates $(41,42)$. It is particularly indicated for surgeons with limited experience and in cases where misidentification and $\mathrm{FN}$ rates are known to be higher (neoadjuvant therapy, prior breast/axillary surgery, obese patients and when the use of BD or radioactive colloid alone fails to produce a signal in the axilla) $(43,44)$. In the dual technique the BD helps with localization post-incision, and LNs that are radioactive, blue, or both are recognised as SLNs (45).

Lymphoscintigraphy is not suggested to be used as routine but only in the cases at higher risk of failing the SLN identification (i.e., previous breast or axillary surgery) (46).

In a meta-analysis of more than 8,000 patients from a systematic review of 69 trials about SLNB, the identification rate of these nodes was $96 \%$, with a $\mathrm{FN}$ rate of $7.3 \%$ (range $0 \%$ to $29 \%$ ) (47). The identification rate with the dual technique was $97 \%$ in the AMAROS trial among 1953 patients with an operable invasive T1-2 BC without clinical suspect regional LNs (48), and $99.1 \%$ in patients in the SENTINA trial with advanced disease undergoing SLNB before commencing neoadjuvant chemotherapy (49). In the ALMANAC trial, the dual technique identified SLNs in $96 \%$ of patients, but only $85.6 \%$ with RI or BD alone (50). In the NSABP B-32 trial, the SLNs were identified in more than $97 \%$ of patients (51). Most of the SLNs were both hot and blue (65\%), while $24 \%$ were hot only, $5 \%$ were blue only, and $3.9 \%$ were neither hot nor blue, but palpably abnormal (this can be explained since gross tumor involvement interferes with the uptake of both radiocolloid and $\mathrm{BD}$, and lymph flow is diverted to a node other than the true SLN. That's why all axillary palpably abnormal LNs should always be removed). Removal of two SLNs rather than one almost halved the $\mathrm{FN}$ rate: in fact the $\mathrm{FN}$ rate in this study was $9.8 \%$ and was related to the number of SLNs removed: it was $17.7 \%$ when only one node was removed, $10 \%$ for two nodes, $6.9 \%$ for three nodes, and $5.5 \%$ for four nodes. This trial also showed no significant difference in overall and disease-free survival between those patients undergoing SLNB followed by automatic ALND and those undergoing SLNB followed by ALND only if the SLNs were identified as positive (6). In a systematic review by the American Society of Clinical Oncology, the use of both $\mathrm{BD}$ and RI was associated with an almost significant trend toward fewer $\mathrm{FN}$ results (7\% vs. 9.9\%) (42).

James et al. (10) particularly stressed the importance of surgeon experience in the accuracy of SLN identification. This was also confirmed by a recent multicenter trial (52). After five training cases, the success rates for individual surgeons identifying a SLN ranged from $79 \%$ to $98 \%$. Furthermore, Cox et al. showed that surgeons who performed more than six SLNBs per month had lower failure rates (53). Proper surgical technique in SLNB influences outcomes and minimizes the risk of understaging and undertreating patients (10).

The constraints of the existing combined SLNB technique have led to the development of alternative methods which will be the object of the following description and review.

\section{New techniques and future perspectives in SLNB}

In recent years new techniques for SLNB have been successfully developed. They use innovative tracers such as indocyanine green (ICG), superparamagnetic iron oxide (SPIO), and microbubbles. Whilst each technique has its own advantages/disadvantages (Figure 1), they have shown promising but variable results between studies, small patient numbers and short patient follow-up (45). So they have to be considered still investigational until there is a final evidence that they are accurate in SLNB with a low FN rate.

\section{Indocyanine green (ICG)}

ICG is a FDA approved, low molecular weight organic molecule, which fluoresces in the near infrared (NIR) part of the spectrum where tissue absorption of light is minimal and has a well-established safety profile. ICG was initially 


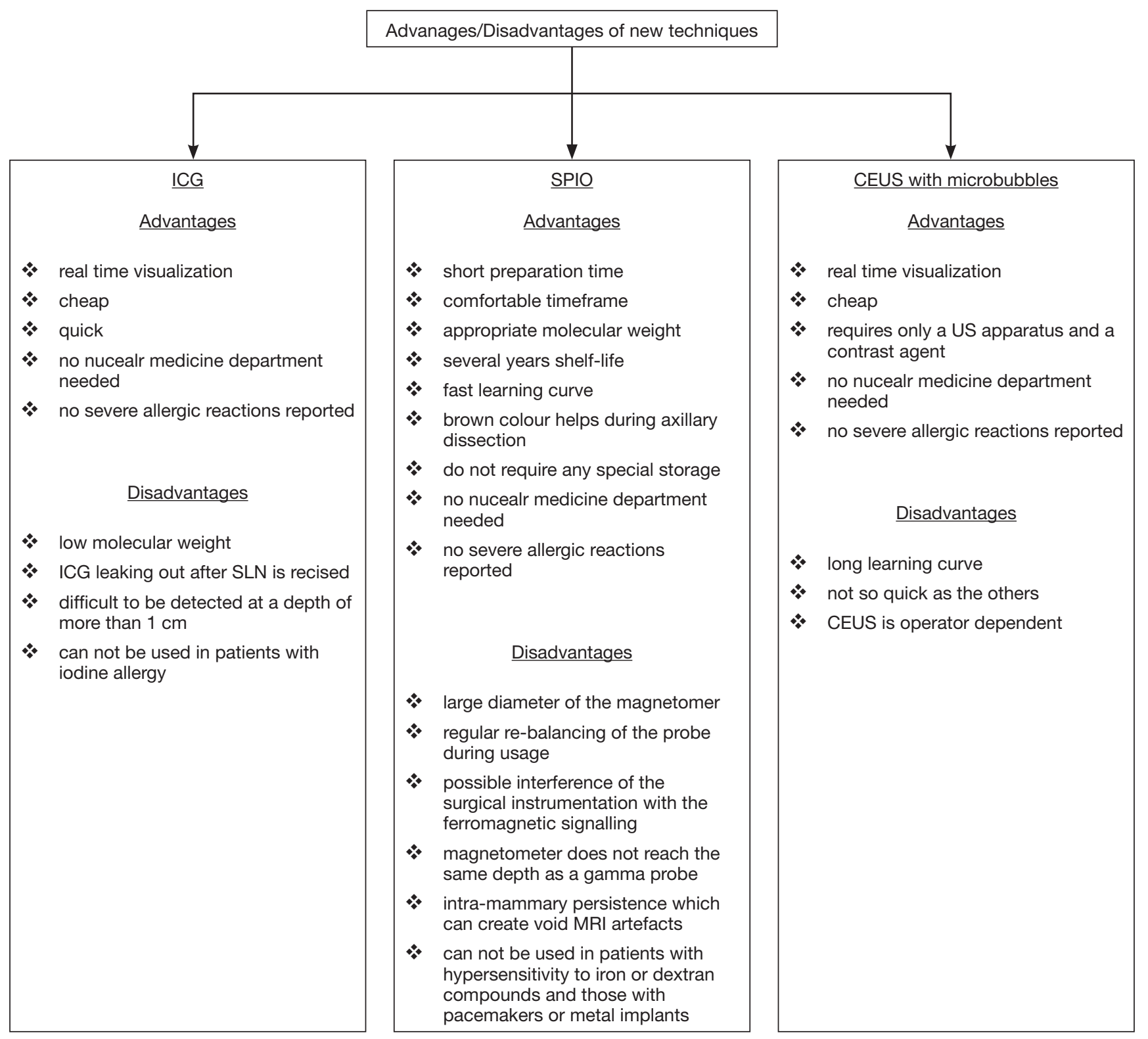

Figure 1 Advantages/disadvantages of new SLNB techniques in BC. ICG, Indocyanine green; SPIO, superparamagnetic iron oxide; CEUS, contrast enhanced ultrasound; SLN, sentinel lymph node; US, ultrasound; SLNB, sentinel lymph node biopsy; BC, breast cancer.

advanced as a visible dye marker in the detection of SLNs, and as such had comparable success to conventional dyes (74\% DR in BC) (54). With improvements to image capturing technology (such as sensitive infrared cameras, the photodynamic eye-PDE or the newer Fluorescence Assisted Resection and Exploration-FLARE imaging system), the use of ICG as a NIR fluorophore improved the DR to approximately 95\% (55). The fluorescence signal is captured by the PDE using a light-emitting diode producing light at a wavelength of $760 \mathrm{~nm}$ in its active state. The detector is a charge-coupled device camera that filters out wavelengths below $820 \mathrm{~nm}$. This NIR fluorescence imaging system visualizes subcutaneous lymphatic flow in real time, as a real time lymphography, and navigates the surgeon to enable an orderly and sequential dissection of SLN.

ICG is injected directly into the breast. The injection site is hugely variable according to the different reported experiences. The majority of studies use periareolar or 
retroareolar subdermal injection, even though ultrasoundguided injection of ICG near to and surrounding ultrasound-identified SLN has been reported (45). Samorani et al. described a protocol of subareolar injection for multicentric BC and intradermal injection for unicentric $\mathrm{BC}$ (56). The injected quantity may depend on the surgeon preferences, the breast volume and the BMI of the patient. A recent meta-analysis showed as ICG injection with reduced concentration $(<5 \mathrm{mg} / \mathrm{mL})$ and larger volume $(\geq 2 \mathrm{~mL})$ may increase sensitivity and DR (57). A quick breast massage should follow the injection. The time between the injection and the incision varies but it should happen by $10 \mathrm{~min}$. The SLNs are then localized using a fluorescent imaging system (56-58): the tracer progress is followed through the lymphatic ducts to the SLN using an excitation illumination system in combination with a high sensitivity camera, which detects the emitted fluorescence. The axillary skin incision should be made only when the tracer has visibly reached the axilla, not before, otherwise, by interrupting the vessels too soon, the detection of SLNs becomes much more difficult (56). ICG fluorescence is scattered by superficial tissues and is difficult to be detected at a depth of more than $1 \mathrm{~cm}$. Thus caution is required when searching for SLNs at lower depths particularly in obese patients.

A major advantage of ICG is that it enables real time visualization of lymph flows from the breast to the axilla. Thus, SLNs can be identified and resected more rapidly and easily, especially in cases with multiple lymph drainage pathways, where ICG can detect multiple SLNs. ICG is reported to be much cheaper compared with a radiotracer. The fluorescence imaging device is also cheaper than a gamma probe. Five milligrams of ICG costs about $\$ 1.50$, while a radiotracer is about \$200 in Japan (59). Therefore, as a result of the lower cost, patients in developing countries may also benefit from ICG tracer. Moreover, the involvement of a nuclear medicine department is not necessary and there is no exposition to radiations. On the other hand, it has been observed a higher number of SLNs identified with ICG, probability due to the low molecular weight which translate into rapid migration in the lymphatics spreading beyond the SLN to secondary draining LNs (55). In their review Ahmed et al. (45) reported a mean number of nodes excised between 3 and 5.4 for ICG and between 1 and 2.4 for BD. ICG probably travels to higher echelon nodes than $\mathrm{BD}$ does, resulting in unnecessarily excessive dissection and removal of nodes.

Furthermore, when the first SLN is resected, lymph vessels are cut and ICG leaks out. The leaked ICG spreads to the surgical field, making it difficult to detect another fluorescent node. This problem can be solved by reducing the amount of ICG. Lastly ICG cannot be used in patients with iodine allergy, because it contains iodine.

Since ICG fluorescence is visualized by collecting NIR rays in dimmed light conditions, recently Toh et al. developed a new promising ICG imaging system called HperEye, which allows the transcutaneous visualization of lymphatic vessels under normal light conditions, thus facilitating the identification and detection of SLNs without affecting the surgical procedure, together with a high sensitivity and specificity (60).

Ahmed et al. found that ICG was significantly better than BD in terms of improved SLN identification, while there was no statistically significant difference between ICG and RI although almost all showed SLN identification to be higher with ICG. No difference was noticed also between ICG and the dual technique. They reported a SLN identification rate between $93.1 \%$ and $100 \%$ (in $11 / 15$ studies) of patients (45).

Increasing clinical results for the ICG method support a higher SLN DR (99-100\%) compared with the use of BD $(61,62)$. Moreover, several clinical trials demonstrated that ICG method is safe and achieves a high SLN DR that is comparable or superior to the RI method (56,63-65). Sugie et al. reported a similar overall DR of SLNs between ICG and RI with a significant improvement achieved by the combination of methods compared with RI alone (66). Tong et al. showed that a combined ICG and BD tracer technique was superior to the use of BD alone for identifying SLNs with higher sensitivity, and for predicting axillary LN status in patients with $\mathrm{BC}$; in addition, the combined technique had reduced FN results (67). Similar results were reported by Hirano et al. in a large series of patients (59). Samorani et al. reported their experience in the use of ICG only for SLNB and they demonstrated that there are no advantages in the association of ICG with BD, as the use of ICG only, once the technique is mastered, allows, always and in every case, the removal of the SLN with a $100 \%$ DR (68). On the other hand, Ji et al. affirmed that the efficiency and sensitivity of SLNB can be improved by combining ICG with $\mathrm{BD}$ since the use of blue tracer significantly decreased the average time to detect each SLN, and it increased the number of SLNs identified (69).

To confirm the importance of this upcoming technique a recent meta-analysis by Zhang et al. reported that ICG guided SLNB had a 98\% DR; the pooled sensitivity (0.92) and specificity (1) were relatively high and the $\mathrm{FN}$ rate was 
relatively low (8\%) going down to $4 \%$ when when ICG was combined with $\mathrm{BD}$; in the presence of metastases, the diagnostic performance of this method was good, with relatively high sensitivity and very high specificity (70).

The last published meta-analysis on this topic of 12 non-randomized comparative studies, reported that ICG was equal to or better than RI in localizing SLNs and tumor-positive SLNs. These results confirm that the ICG fluorescence method is a useful alternative to the standard RI method for SLNB (71).

No severe adverse events directly related to the ICG dye have been reported. Recent improvements in this technique, combined with its good safety profile, made the use of ICG to find SLNs an attractive and promising alternative for SLNB in BC. However, a good standardisation of the technique between studies is low, which makes it difficult to reliably draw final conclusions. This shows how this technique is still in development with significant refinement necessary (45).

\section{Superparamagnetic iron oxide (SPIO)}

SPIO is a non-invasive magnetic tracer for SLNB detected by a handheld magnetometer. The SentiMAG multicentre trial (72) was the first trial that evaluated this new magnetic technique for SLNB against the standard one. When injected subcutaneously, SPIO moves into SLNs within minutes, and iron deposition is seen predominantly within sinuses and in macrophages. In the event of metastatic involvement of the node, SPIOs are seen to deposit within uninvolved areas of the node only (73). The nodes can be visualized on magnetic resonance imaging (MRI) and at operation are often colored brown or black (74). The SentiMAG trial group developed 2 CE marked devices: an injectable magnetic tracer (Sienna+) and a handheld magnetometer (SentiMag) that generates an alternating magnetic field which temporarily magnetizes the SPIO and senses the particles' magnetic response. The particle diameter of Sienna+, including its organic coating, is $60 \mathrm{~nm}$. It is ideally suited for SLNB since this diameter enables the SLNs to selectively filter out the particles and is similar to the particle size of standard RI tracers. The diameter of the magnetometer is slightly larger $(6 \mathrm{~mm})$ than that of the gamma probe. The magnetic technique was well standardized. A $5 \mathrm{~mL}$ periareolar subcutaneous injection was administered, consisting of $2 \mathrm{~mL}$ of magnetic tracer (Siennat) diluted with $3 \mathrm{~mL}$ of normal saline. This was injected intraoperatively (after induction of anesthesia) followed by a 5 min massage. The median Sienna+ migration time before surgery was $20 \mathrm{~min}$. The surgeon used the handheld magnetometer for the SLN localization. All SLNs detected intraoperatively by using the handheld magnetometer or gamma probe or nodes that were blue or black, were excised. All metal retractors were removed from the surgical field while the magnetometer was used. Excision of nodes identified by magnetometer followed the same "10\% rule" used for the gamma probe. Any palpable nodes were also removed. They reported no difference in identification rate $(95 \%$ with the standard technique and $94.4 \%$ with the magnetic technique) and no difference in the average number of LN removed (1.9 vs. 2.0 per patient) (72).

Similar results were achieved by the Central-European SentiMag study (75) reporting similar DRs between the standard RI technique and the magnetic one $(97.3 \%$ vs. 98.0\%), with a similar average number of removed SLNs per patient and a higher per patient malignancy DR for the SPIO tracer.

In agreement with the previous experiences Rubio et al. showed no difference in DR between the standard RI technique and the magnetic one $(95.7 \%$ vs. $98.3 \%)$ with a significant higher average number of SLN excised in the SPIO group (76).

The "IMAGINE" Spanish multicentre study reported similar DRs between RI technique and magnetic technique with the ex-vivo and intraoperative DRs at the node level slightly higher for the magnetic technique. The SPIO tracer also showed a higher per patient malignancy DR (77).

The French Sentimag Feasibility Trial evaluated the magnetic technique in comparison to the standard technique (RI with or without BD) (78). The DR was $97.2 \%$ for Sienna+ and $95.4 \%$ for the standard technique with more SLNs detected with Sienna+ (97.2\% vs. 90.2\%). Furthermore, the $\mathrm{N}+$ status does not seem to be an obstacle for the magnetic method which shows a tendency to identify more involved SLNs, a trend already described by Thill et al. (75).

A meta-analysis of five clinical trials comparing Sienna+ to a standard technique confirmed that Sienna+ is noninferior with respect to SLN identification per patient, as well as per SLN. Importantly, non-inferiority was also shown with respect to the ability to accurately identify patients with malignant SLNs (79).

In another recent meta-analysis of seven randomized trials by Zada et al., the SPIO technique was not inferior to the standard technique in identification rate $(97.1 \% \mathrm{vs}$. 96.8\%), total LNs retrieved (1.9 vs. 1.8 nodes per patient), and $\mathrm{FN}$ rate $(8.4 \%$ vs. $10.9 \%)$. There was a trend towards 
a lower $\mathrm{FN}$ rate in favour of the magnetic technique, but this was not statistically significant. The total number of LNs retrieved was significantly higher with the magnetic technique. The mean discordance rate between the two techniques was $3.9 \%$ (range $1.7 \%$ to $6.9 \%$ ) (80).

The SentiMag technique offers many advantages: surgeon can inject the magnetic tracer directly in the operation room, the preparation time is much shorter than for the RI, provides a very comfortable timeframe and the SPIO tracer is well retained in the "true" SLN. The shelflife of the magnetic tracer is several years and the learning curve seems to be very fast, especially if compared to the other techniques. The brown color does not seem to be sufficient to make identification alone but can help the surgeon during the axillary dissection. Sienna+ particles do not require any special storage and there are no radiation risks or any of the legislative controls associated with the handling of isotopes, neither for healthcare personnel nor for the patient. On the other hand, the diameter of the magnetometer is quite larger than the RI probe, even though a smaller one has been recently commercialized. Moreover, this technique requires a regular re-balancing of the probe during usage before each signal acquisition. Another technical limitation is a possible interference of the surgical instrumentation with the ferromagnetic signalling. To prevent it, the use of plastic surgical material is encouraged while the measurement with SentiMg is performed. Particular attention should be given to the intra-mammary persistence of Sienna+ which can create void artefacts and could hamper the interpretation of a postoperative breast MRI obscuring important clinical findings. Subareolar injections may have a higher risk of void artefacts than intratumoral or peritumoral injections of small magnetic tracer volumes, because the tumour area is excised (80). In a small study of ten MRI scans performed in six patients, void artifacts were noted at the subareolar injection site in all scans and all artifacts were greater than $5 \mathrm{~mm}$ in greatest dimension. Based on current evidence, the magnetic technique should not be used in patients undergoing SLNB before primary chemotherapy (if MRI is used to assess response to treatment), those undergoing breast MRI surveillance (such as BRCA mutation carriers) or those with a mammographically occult tumour at diagnosis, in whom MRI may be required for further assessment or follow-up imaging (81). More research should clarify the patient cohort eligible for the magnetic technique and optimize the amount of magnetic tracer required. Another concern is that the magnetometer does not reach the same depth as a gamma probe, which can have consequences for the identification of deeper nodes, as demonstrated in the recently published MELAMAG Trial (82). Patients with hypersensitivity to iron or dextran compounds and those with pacemakers or metal implants were excluded from the clinical trials and would not be candidates for using Sienna+ for SLN mapping. A dermopigmentation is the most frequent complication reported at a rate up to $20 \%$ in correspondence of the injection site, which usually vanished over time $(76,78)$. No severe allergic reactions were reported in any of the trials and meta-analysis reviewed.

All these encouraging results led to consider magnetic technique as feasible, but its performance should now be evaluated in a large randomized non-commercial controlled trial before clinical implementation (80).

\section{SPIO enhanced MRI}

Motomura et al. evaluated SPIO enhanced MRI for the detection of metastases in SLNs of patients with BC. A node was considered involved if the entire node or a focal area did not show low signal intensity on MRI. The sensitivity, specificity, and overall accuracy of MRI for the diagnosis of SLN metastases were $84 \%, 91 \%$, and $89 \%$, respectively (74). A more recent update by the same group introduced a SPIO enhanced MRI at $3 \mathrm{~T}$ (83). They reported much better results: On a patient-by-patient basis, the sensitivity, specificity and accuracy of MRI for the diagnosis of SLN metastases were $100 \%, 96 \%$ and $97 \%$. Authors concluded that SLNB may be avoided in patients with BC who have non-metastatic SLNs on SPIO-MRI.

The SentiMag multicentre trial imaging subprotocol evaluated the use of SPIO MRI for pre-operative localization of SLN and its potential for non-invasive identification of SLNs metastases (84). SPIO contrast agents are known to provide "negative contrast" on T2 weighted MRI. SPIOs are injected interstitially, they are taken up by SLNs, and therefore SPIO MRI can be used as an alternative for pre-operative localization of SLNs which are visualized as a drop of signal intensity. LNs showing inhomogeneous SPIO uptake were classified as metastatic. SPIO-MRI successfully identified SLNs in 91\% of patients. One patient had metastatic involvement of four LNs, and this was identified in one node on preoperative MRI. Two false positive cases were reported. The concordance between the number of nodes identified by imaging and during surgery was $55 \%$ for both the magnetic and the 
combined technique. So it seems that SPIO MRI is a feasible technique for preoperative localization of SLNs and, in combination with intraoperative use of a handheld magnetometer, provides an entirely RI-free technique for SLNB. It gives the surgeon a detailed anatomical information on the location of the SLNs, which serves as a detailed surgical roadmap. Moreover, a noninvasive method that accurately diagnoses SLN metastases would prevent patients undergoing unnecessary SLNB. However further optimization of the technique and research with larger series of patients is needed.

Li et al. evaluated magnetic resonance lymphography with gadolinium (Gd-MRI) in SLN identification and metastasis detection in patients with BC (85). There was a significant correlation between the SLN numbers found by Gd-MRI and BD methods. Using BD as the gold standard, the sensitivity of Gd-MRL was $95.65 \%$ and the $\mathrm{FN}$ rate was $4.3 \%$ for axillary lymphatic metastasis detection. With heterogeneous enhancement and enhancement defect as the diagnostic criteria, Gd-MRL gave a sensitivity of $89.29 \%$ and specificity of $89.66 \%$ in discriminating malignant from benign SLNs.

Compared with SPIO-MRL, Gd-MRL is cheaper and more convenient. SPIO is a negative contrast and thus cannot image the lymph vessel. Compared with iopamidol-CT lymphography, Gd-MRL lacks radiation exposure, possibility of anaphylactic shock and nephrotoxic impairment (86). The good results achieved by this technique suggest a potential value in a future clinical practice.

\section{Contrast enhanced ultrasound (CEUS) with microbubbles}

This is an innovative technique where microbubble contrast agent, based on the use of dispersion with sulfur hexafluoride gas, is injected intradermally around the areola. Breast lymphatics are then visualized by CEUS and followed to identify and biopsy SLNs (87).

Sever et al. tried to standardize a protocol (88). They performed a periareolar intradermal injection of $0.2-0.5 \mathrm{~mL}$ phospholipid-stabilised microbubbles containing sulphur hexafluoride gas with a mean diameter of $2.5 \mu \mathrm{m}$ (SonovueBracco) reconstituted with $2 \mathrm{~mL}$ sterile saline. The breast was massaged for 10-30 s and lymphatic channels were visualised immediately on contrast pulse sequencing and followed into the axilla. The transit time from injection to arrival in the axillary nodes was $15-45 \mathrm{~s}$. Areas of contrast accumulation were imaged with greyscale or live dual images to confirm the presence of an architecturally defined LN. The CEUS-identified SLNs were then localized with guidewires before undergoing SLNB with the standard dual technique. It resulted in SLN identification in $89 \%$ of patients (89). In a larger series by Cox et al. the technique failed to visualise or successfully biopsy $13.3 \%$ of patients, identifying positive SLN in $87.7 \%$. There were 22 falsenegative results, which meant that sensitivity was $61 \%$ (low compared to the dual technique) and specificity $100 \%$ (87).

In the review by Ahmed et al. the standard dual technique was significantly better than CEUS in terms of SLN identification rate which anyway was between $87.7 \%$ and $89 \%(45)$.

A recent meta-analysis of five studies reported the SLN identification rate ranging from $9.3 \%$ to $55.2 \%$, the sensitivity from $61 \%$ to $89 \%$, the $\mathrm{FN}$ rate from $6.6 \%$ to $39 \%$ and the presence of micro/macrometastases from $1.9 \%$ to $64.3 \%(90)$.

Better results were achieved by Xie $e t$ al. who studied CEUS with Sonovue intradermal subareolar injection to localize SLN and the value of enhancing patterns in diagnosing SLN metastases (91). They showed no significant difference in SLN identification rate between CEUS and BD (97\% vs. 96\%). CEUS method detected less SLNs than BD while the positive rates of SLNs identified by CEUS was significantly higher than that by BD. Sensitivity of predicting SLNs metastases by CEUS enhancing pattern was $81.8 \%$, the specificity was $86.2 \%$, and the accuracy was $84.7 \%$. They also described three patterns of enhancement of SLNs and the disomogeneous one was more common in involved SLNs.

In a preliminary clinical trial by Matsuzawa et al., CEUS was performed with a subareolar contrast injection to evaluate the SLN detection and intravenous contrast injection studied at color doppler to evaluate the presence of metastases in SLN, visualizing their microvascularities (92). The peripheral vascularization pattern was more frequently found in metastatic LNs. They used Sonazoid as contrast agent, a perflubutane microbubble that is stabilized using a phospholipid. Their results were very promising: sensitivity $81.8 \%$, specificity $95.2 \%$, positive predictive value $90.0 \%$, negative predictive value $90.9 \%$, accuracy $90.6 \%$.

Dellaportas et al. reported their experience with CEUS using totally intravenous contrast injection for a preoperative detection of malignant SLN (93). They showed a negative predictive value of $90 \%$ and positive predictive value of $75 \%$. Overall sensitivity was $83.33 \%$ and specificity was $84.38 \%$. Multivariate analysis showed 
that the CEUS outcome was only correlated with the actual final histopathological report on the SLN. All the cases with 3 or more positive LNs were detected preoperatively with CEUS. They observed that involved nodes enhanced heterogeneously, with an early wash-in and wash-out enhancement.

Last meta-analysis published on this topic by Moody et al. evaluated whether CEUS-guided core biopsy of SLN could identify metastatic nodes preoperatively reducing the number of surgical SLNBs and whether CEUS SLN identification and localization is a viable alternative to standard lymphatic mapping using RI and BD (94). The SLN identification and localization rate for CEUS-guided skin marking was $70-100 \%$ and for CEUS guided-wire localization was $89-97 \%$. Across the four studies that evaluated preoperative CEUS-guided SLNB, pooled sensitivity for identification of nodal metastases was $54 \%$ and pooled specificity $100 \%$; the FNR ranged from $8 \%$ to $17 \%$.

One advantage of this method is the real-time imaging for SLNs. It requires only a US apparatus and a contrast agent readily available on market so it is cheap and does not need radioactive materials. The patient is not exposed to radiation and the method is not invasive. CEUS guided core biopsy could potentially decrease the number of SLNBs and ALNDs in women with BC. CEUS has been proved to be accurate to identify and localize the SLNs preoperatively with SLN identification rates comparable to standard lymphatic mapping with RI and BD. So CEUS-guided SLN localizations may offer a viable alternative for developing countries that may not have access to nuclear medicine facilities. There are no iodine and proteins in sulfur hexafluoride microbubbles, which prevents patients from allergy. Indeed there have been reported no significant side effects or complications from the injection of microbubbles.

On the other hand, the learning curve for this technique seems to be quite long and the US still remains operator dependent.

Even though it is a promising technique both for SLNs identification and for metastasizing SLNs detection, further studies are necessary to well standardize the method and improve the sensitivity and specificity. Randomized control trials with large series of patients are suggested in order to compare this technique with the current techniques used for SLN detection.

\section{Conclusions}

SLNB is still a stronghold in the management of early
$\mathrm{BC}$ since the status of the axillary LNs remains one of the most important prognostic factors. Although the standard dual technique has reached a good level of standardization and accuracy it is burdened by several drawbacks. The use of RI creates logistical challenges for hospitals, legislative requirements, exposition to radiations both for the patient and for the healthcare staff. Moreover, the $6 \mathrm{~h}$ half-life of the isotope restricts scheduling of surgery because the injection is done by the nuclear medicine department. The development of new techniques in SLNB together with improvement in surgical strategies are clouding the traditional methods. Incoming experiences in endoscopic SLNB and ALND have been described, reporting low complication rates, better cosmetic results and similar efficacy to the traditional open axillary surgery (95). The new SLNB techniques seem to be safe, feasible and have shown very high improvements in accuracy, sensitivity and specificity in last years. It would be time to develop new comparative studies between the new techniques themselves to better clarify their value and efficacy. Anyway, all the last evidences show similar results or better than the traditional ones. Surgeon can be independent from the nuclear medicine department and can manage the operating independently. However, it should be well clarified how easy the availability of the new devices is and their relative cost.

Since the new methods have now achieved a high qualitative standard and seem to be totally competitive with the traditional ones, randomized controlled trials are needed to assess their outcomes against the standard dual technique and be fully approved as new standard of care for SLNB.

\section{Acknowledgments}

Funding: None.

\section{Footnote}

Provenance and Peer Review: This article was commissioned by the editorial office, Translational Cancer Research for the series "Update of Current Evidences in Breast Cancer Multidisciplinary Management". The article has undergone external peer review.

Conflicts of Interest: All authors have completed the ICMJE uniform disclosure form (available at http://dx.doi. org/10.21037/tcr.2018.02.07). The series "Update of Current Evidences in Breast Cancer Multidisciplinary Management" was commissioned by the editorial office 
without any funding or sponsorship. GF served as the unpaid Guest Editor of the series and serves as an unpaid editorial board member of Translational Cancer Research from Nov 2016 to Dec 2018. The authors have no other conflicts of interest to declare.

Ethical Statement: The authors are accountable for all aspects of the work in ensuring that questions related to the accuracy or integrity of any part of the work are appropriately investigated and resolved.

Open Access Statement: This is an Open Access article distributed in accordance with the Creative Commons Attribution-NonCommercial-NoDerivs 4.0 International License (CC BY-NC-ND 4.0), which permits the noncommercial replication and distribution of the article with the strict proviso that no changes or edits are made and the original work is properly cited (including links to both the formal publication through the relevant DOI and the license). See: https://creativecommons.org/licenses/by-nc-nd/4.0/.

\section{References}

1. Robinson DS, Sample WF, Fee HJ, et al. Regional lymphatic drainage in primary malignant melanoma of the trunk determined by colloidal gold scanning. Surg Forum 1977;28:147-8.

2. Giuliano AE, Kirgan DM, Guenther JM, et al. Lymphatic mapping and sentinel lymphadenectomy for breast cancer. Ann Surg 1994;220:391.

3. Krag DN, Weaver DL, Alex JC, et al. Surgical resection and radiolocalization of the sentinel lymph node in breast cancer using a gamma probe. Surg Oncol 1993;2:335.

4. Giuliano AE, Jones RC, Brennan M, et al. Sentinel lymphadenectomy in breast cancer. J Clin Oncol 1997;15:2345-50.

5. Lyman GH, Somerfield MR, Bosserman LD, et al. Sentinel lymph node biopsy for patients with early-stage breast cancer: american society of clinical oncology clinical practice guideline update. J Clin Oncol 2017;35:561-4.

6. Krag DN, Anderson SJ, Julian TB, et al. Sentinel-lymphnode resection compared with conventional axillary-lymphnode dissection in clinically node-negative patients with breast cancer: Overall survival findings from the NSABP B-32 randomised phase 3 trial. Lancet Oncol 2010;11:927-33.

7. Veronesi U, Paganelli G, Viale G, et al. A randomized comparison of sentinel-node biopsy with routine axillary dissection in breast cancer. N Engl J Med 2003;349:546-53.
8. Veronesi U, Viale G, Paganelli G, et al. Sentinel lymph node biopsy in breast cancer: Ten-year results of a randomized controlled study. Ann Surg 2010;251:595-600.

9. Rao R, Euhus D, Mayo HG, et al. Axillary node interventions in breast cancer: a systematic review. JAMA 2013;310:1385.

10. James TA, Coffman AR, Chagpar AB, et al. Troubleshooting sentinel lymph node biopsy in breast cancer surgery. Ann Surg Oncol 2016;23:3459.

11. Barthelmes L, Goyal A, Newcombe RG, McNeill F, Mansel RE, NEW START and ALMANAC Study Groups. Adverse reactions to patent blue $\mathrm{V}$ dye: the NEW START and ALMANAC experience. Eur J Surg Oncol 2010;36:399-403.

12. Raut CP, Hunt KK, Akins JS, et al. Incidence of anaphylactoid reactions to isosulfan blue dye during breast carcinoma lymphatic mapping in patients treated with preoperative prophylaxis: results of a surgical prospective clinical practice protocol. Cancer 2005;104:692.

13. Wilke LG, McCall LM, Posther KE, et al. Surgical complications associated with sentinel lymph node biopsy: results from a prospective international cooperative group trial. Ann Surg Oncol 2006;13:491.

14. Thevarajah S, Huston TL, Simmons RM. A comparison of the adverse reactions associated with isosulfan blue versus methylene blue dye in sentinel lymph node biopsy for breast cancer. Am J Surg 2005;189:236.

15. Bleicher RJ, Kloth DD, Robinson D, et al. Inflammatory cutaneous adverse effects of methylene blue dye injection for lymphatic mapping/sentinel lymphadenectomy. J Surg Oncol 2009;99:356.

16. Teknos D, Ramcharan A, Oluwole SF. Pulmonary edema associated with methylene blue dye administration during sentinel lymph node biopsy. J Natl Med Assoc 2008;100:1483.

17. Zakaria S, Hoskin TL, Degnim AC. Safety and technical success of methylene blue dye for lymphatic mapping in breast cancer. Am J Surg 2008;196:228.

18. Chagpar AB, Martin RC, Scoggins CR, et al. Factors predicting failure to identify a sentinel lymph node in breast cancer. Surgery 2005;138:56.

19. Blessing WD, Stolier AJ, Teng SC, et al. A comparison of methylene blue and lymphazurin in breast cancer sentinel node mapping. Am J Surg 2002;184:341-5.

20. Masannat Y, Shenoy H, Speirs V, et al. Properties and characteristics of the dyes injected to assist axillary sentinel node localization in breast surgery. Eur J Surg Oncol 2006;32:381-4.

21. Varghese P, Abdel-Rahman AT, Akberali S, et al. 
Methylene blue dye: a safe and effective alternative for sentinel lymph node localization. Breast J 2008;14:61-7.

22. Simmons R, Thevarajah S, Brennan MB, et al. Methylene blue dye as an alternative to isosulfan blue dye for sentinel lymph node localization. Ann Surg Oncol 2003;10:242-7.

23. Gropper AB, Calvillo KZ, Dominici L, et al. Sentinel lymph node biopsy in pregnant women with breast cancer. Ann Surg Oncol 2014;21:2506-11.

24. Paulinelli RR, Freitas-Junior R, Rahal RM, et al. A prospective randomized trial comparing patent blue and methylene blue for the detection of the sentinel lymph node in breast cancer patients. Rev Assoc Med Bras (1992) 2017;63:118-23.

25. Krikanova M, Biggar M, Moss D. Accuracy of sentinel node biopsy for breast cancer using blue dye alone. Breast J 2010;16:384-8

26. McMasters KM, Wong SL, Martin RC 2nd, et al. Dermal injection of radioactive colloid is superior to peritumoral injection for breast cancer sentinel lymph node biopsy: results of a multiinstitutional study. Ann Surg 2001;233:676.

27. Povoski SP, Olsen JO, Young DC, et al. Prospective randomized clinical trial comparing intradermal, intraparenchymal, and subareolar injection routes for sentinel lymph node mapping and biopsy in breast cancer. Ann Surg Oncol 2006;13:1412.

28. Motomura K, Komoike Y, Hasegawa Y, et al. Intradermal radioisotope injection is superior to subdermal injection for the identification of the sentinel node in breast cancer patients. J Surg Oncol 2003;82:91.

29. Berrocal J, Saperstein L, Grube B, et al. Intraoperative injection of technetium-99m sulfur colloid for sentinel lymph node biopsy in breast cancer patients: a single institution experience. Surg Res Pract 2017;2017:5924802.

30. Martin RC 2nd, Edwards MJ, Wong SL, et al. Practical guidelines for optimal gamma probe detection of sentinel lymph nodes in breast cancer: results of a multiinstitutional study. For the University of Louisville Breast Cancer Study Group. Surgery 2000;128:139.

31. Nguyen TK. Magnetic nanoparticles: from fabrication to clinical applications. New York: CRC Press, 2012.

32. Kim CK, Zukotynski KA. Desirable properties of radiopharmaceuticals for sentinel node mapping in patients with breast cancer given the paradigm shift in patient management. Clin Nucl Med 2017;42:275-9.

33. Surasi DS, O'Malley J, Bhambhvani P. 99m TcTilmanocept: a novel molecular agent for lymphatic mapping and sentinel lymph node localization. J Nucl Med Technol 2015;43:87-91.
34. Li N, Wang X, Lin B, et al. Clinical Evaluation of 99mTcRituximab for sentinel lymph node mapping in breast cancer patients. J Nucl Med 2016;57:1214-20.

35. İlem-Özdemir D, Yararbas U, Zengel B, et al. Preparation of $99 \mathrm{~m}$ Tc-isosulfan blue for lymph node localization in rats 99m Tc-isosulfan blue for lymph node localization. Appl Radiat Isot 2016;118:338-42.

36. Veronesi U, Paganelli G, Galimberti V, et al. Sentinel-node biopsy to avoid axillary dissection in breast cancer with clinically negative lymph-nodes. Lancet 1997;349:1864.

37. Crossin JA, Johnson AC, Stewart PB, et al. Gamma-probeguided resection of the sentinel lymph node in breast cancer. Am Surg 1998;64:666.

38. McMasters KM, Tuttle TM, Carlson DJ, et al. Sentinel lymph node biopsy for breast cancer: a suitable alternative to routine axillary dissection in multi institutional practice when optimal technique is used. J Clin Oncol 2000;18:2560.

39. He PS, Li F, Li GH, et al. The combination of blue dye and radioisotope versus radioisotope alone during sentinel lymph node biopsy for breast cancer: a systematic review. BMC Cancer 2016;16:107.

40. Motomura K, Inaji H, Komoike Y, et al. Combination technique is superior to dye alone in identification of the sentinel node in breast cancer. J Surg Oncol 2001;76:95-9.

41. Cody HS 3rd, Fey J, Akhurst T, et al. Complementarity of blue dye and isotope in sentinel node localization for breast cancer: univariate and multivariate analysis of 966 procedures. Ann Surg Oncol 2001;8:13-9.

42. Lyman GH, Giuliano AE, Somerfield MR, et al. American Society of Clinical Oncology guideline recommendations for sentinel lymph node biopsy in early stage breast cancer. J Clin Oncol 2005;23:7703-20.

43. Schwartz GF, Giuliano AE, Veronesi U. Consensus Conference Committee. Proceedings of the Consensus Conference on the role of sentinel lymph node biopsy in carcinoma of the breast, April 19-22, 2001, Philadelphia, Pennsylvania. Cancer 2002;94:2542-51.

44. Boughey JC, Suman VJ, Mittendorf EA, et al. Sentinel lymph node surgery after neoadjuvant chemotherapy in patients with node-positive breast cancer: the ACOSOG Z1071 (Alliance) clinical trial. JAMA 2013;310:1455-61.

45. Ahmed M, Purushotham AD, Douek M. Novel techniques for sentinel lymph node biopsy in breast cancer: a systematic review. Lancet Oncol 2014;15:e351.

46. Uren RF, Howman-Giles R, Chung D, et al. Nuclear medicine aspects of melanoma and breast lymphatic mapping. Semin Oncol 2004;31:338.

47. Kim T, Giuliano AE, Lyman GH. Lymphatic mapping 
and sentinel lymph node biopsy in early-stage breast carcinoma: a meta-analysis. Cancer 2006;106:4-16.

48. Straver ME, Meijnen P, van Tienhoven G, et al. Sentinel node identification rate and nodal involvement in the EORTC 10981-22023 AMAROS trial. Ann Surg Oncol 2010;17:1854-61.

49. Kuehn T, Bauerfeind I, Fehm T, et al. Sentinel-lymphnode biopsy in patients with breast cancer before and after neoadjuvant chemotherapy (SENTINA): a prospective, multicentre cohort study. Lancet Oncol 2013;14:609-18.

50. Goyal A, Newcombe RG, Chhabra A, et al. and the ALMANAC Trialists Group. Factors aff ecting failed localisation and false-negative rates of sentinel node biopsy in breast cancer-results of the ALMANAC validation phase. Breast Cancer Res Treat 2006;99:203-8.

51. Krag DN, Anderson SJ, Julian TB, et al, and the National Surgical Adjuvant Breast and Bowel Project. Technical outcomes of sentinellymph- node resection and conventional axillary-lymph-node dissection in patients with clinically node-negative breast cancer: results from the NSABP B-32 randomised phase III trial. Lancet Oncol 2007;8:881-8.

52. Krag D, Weaver D, Ashikaga T, et al. The sentinel node in breast cancer-a multicenter validation study. N Engl J Med 1998;339:941--6.

53. Cox CE, Salud CJ, Cantor A, et al. Learning curves for breast cancer sentinel lymph node mapping based on surgical volume analysis. J Am Coll Surg 2001;193:593-600.

54. Motomura K, Inaji H, Komoike Y, et al. Sentinel node biopsy guided by indocyanin green dye in breast cancer patients. Jpn J Clin Oncol 1999;29:604-7.

55. Polom K, Murawa D, Rho YS, et al. Current trends and emerging future of indocyanine green usage in surgery and oncology: a literature review. Cancer 2011;117:4812-22.

56. Samorani D, Fogacci T, Panzini I, et al. The use of indocyanine green to detect sentinel nodes in breast cancer: a prospective study. Eur J Surg Oncol 2015;41:64.

57. Xiong L, Gazyakan E, Yang W, et al. Indocyanine green fluorescence-guided sentinel node biopsy: a meta-analysis on detection rate and diagnostic performance. Eur J Surg Oncol 2014;40:843.

58. Pitsinis V, Provenzano E, Kaklamanis L, et al. Indocyanine green fluorescence mapping for sentinel lymph node biopsy in early breast cancer. Surg Oncol 2015;24:375.

59. Hirano A, Kamimura M, Ogura K, et al. A comparison of indocyanine green fluorescence imaging plus blue dye and blue dye alone for sentinel node navigation surgery in breast cancer patients. Ann Surg Oncol 2012;19:4112-6.

60. Toh U, Iwakuma N, Mishima M, et al. Navigation surgery for intraoperative sentinel lymph node detection using
Indocyanine green (ICG) fluorescence real-time imaging in breast cancer. Breast Cancer Res Treat 2015;153:337-44.

61. Tagaya N, Aoyagi H, Nakagawa A, et al. A novel approach for sentinel lymph node identification using fluorescence imaging and image overlay navigation surgery in patients with breast cancer. World J Surg 2011;35:154-8.

62. Sugie T, Sawada T, Tagaya N, et al. Comparison of the indocyanine green fluorescence and blue dye methods in detection of sentinel lymph nodes in early-stage breast cancer. Ann Surg Oncol 2013;20:2213-8.

63. van der Vorst JR, Schaafsma BE, Verbeek FP, et al. Randomized comparison of near-infrared fluorescence imaging using indocyanine green and 99mtechnetium with or without patent blue for the sentinel lymph node procedure in breast cancer patients. Ann Surg Oncol 2012;19:4104-11.

64. Schaafsma BE, Verbeek FP, Riebergen DD, et al. Clinical trial of combined radio- and fluorescence-guided sentinel lymph node biopsy in breast cancer. Br J Surg 2013;100:1037-44.

65. Ballardini B, Santoro L, Sangalli C, et al. The indocyanine green method is equivalent to the $99 \mathrm{~m}$ Tc-labeled radiotracer method for identifying the sentinel node in breast cancer: a concordance and validation study. Eur J Surg Oncol 2013;39:1332-6.

66. Sugie T, Kinoshita T, Masuda N, et al. Evaluation of the clinical utility of the icg fluorescence method compared with the radioisotope method for sentinel lymph node biopsy in breast cancer. Ann Surg Oncol 2016;23:44-50.

67. Tong M, Guo W, Gao W. Use of fluorescence imaging in combination with patent blue dye versus patent blue dye alone in sentinel lymph node biopsy in breast cancer. J Breast Cancer 2014;17:250-5.

68. Samorani D, Fogacci T, Dellachiesa L. Use of indocyanine green alone for sentinel node biopsy in breast cancer. Breast Cancer 2015;22:442-3.

69. Ji Y, Luo N, Jiang Y, et al. Clinical utility of the additional use of blue dye for indocyanine green for sentinel node biopsy in breast cancer. J Surg Res 2017;215:88-92.

70. Zhang X, Li Y, Zhou Y. Diagnostic performance of indocyanine green-guided sentinel lymph node biopsy in breast cancer: a meta-analysis. PLoS One 2016;11:e0155597.

71. Sugie T, Ikeda T, Kawaguchi A, et al. Sentinel lymph node biopsy using indocyanine green fluorescence in early-stage breast cancer: a meta-analysis. Int J Clin Oncol 2017;22:11.

72. Douek M, Klaase J, Monypenny I, et al. Sentinel node biopsy using a magnetic tracer versus standard technique: the SentiMAG Multicentre Trial. Ann Surg Oncol 2014;21:1237. 
73. Johnson L, Pinder SE, Douek M. Deposition of superparamagnetic iron-oxide nanoparticles in axillary sentinel lymph nodes following subcutaneous injection. Histopathology 2013;62:481-6.

74. Motomura K, Ishitobi M, Komoike Y, et al. SPIOenhanced magnetic resonance imaging for the detection of metastases in sentinel nodes localized by computed tomography lymphography in patients with breast cancer. Ann Surg Oncol 2011;18:3422-9.

75. Thill M, Kurylcio A, Welter R, et al. The CentralEuropean SentiMag study: sentinel lymph node biopsy with superparamagnetic iron oxide (SPIO) vs. radioisotope. Breast 2014;23:175.

76. Rubio IT, Diaz-Botero S, Esgueva A, et al. The superparamagnetic iron oxide is equivalent to the Tc99 radiotracer method for identifying the sentinel lymph node in breast cancer. Eur J Surg Oncol 2015;41:46.

77. Piñero-Madrona A, Torró-Richart JA, de León-Carrillo JM, et al. Superparamagnetic iron oxide as a tracer for sentinel node biopsy in breast cancer: A comparative noninferiority study. Eur J Surg Oncol 2015;41:991-7.

78. Houpeau JL, Chauvet MP, Guillemin F, et al. Sentinel lymph node identification using superparamagnetic iron oxide particles versus radioisotope: The French Sentimag feasibility trial. J Surg Oncol 2016;113:501-7.

79. Teshome M, Wei C, Hunt KK, et al. Use of a magnetic tracer for sentinel lymph node detection in early-stage breast cancer patients: a meta-analysis. Ann Surg Oncol 2016;23:1508-14.

80. Zada A, Peek MC, Ahmed M, et al. Meta-analysis of sentinel lymph node biopsy in breast cancer using the magnetic technique. Br J Surg 2016;103:1409.

81. Huizing E, Anninga B, Young P, et al. Analysis of void artifacts in post-operative breast MRI due to residual SPIO after magnetic SLNB in SentiMAG Trial participants. Eur J Surg Oncol 2015;41:S18.

82. Anninga B, White SH, Moncrieff $M$, et al. MELAMAG Multicentre Trialists Group. Magnetic technique for sentinel lymph node biopsy in melanoma: the MELAMAG Trial. Ann Surg Oncol 2016;23:2070-8.

83. Motomura K, Izumi T, Tateishi S, et al. Superparamagnetic iron oxide-enhanced MRI at $3 \mathrm{~T}$ for accurate axillary staging in breast cancer. Br J Surg 2016;103:60-9.

84. Pouw JJ, Grootendorst MR, Bezooijen R, et al. Preoperative sentinel lymph node localization in breast cancer with superparamagnetic iron oxide MRI: the SentiMAG Multicentre Trial imaging subprotocol. Br J Radiol 2015;88:20150634.

85. Li C, Meng S, Yang X. Sentinel lymph node detection using magnetic resonance lymphography with conventional gadolinium contrast agent in breast cancer: a preliminary clinical study. BMC Cancer 2015;15:213.

86. Shiozawa M, Kobayashi S, Sato Y, et al. Magnetic resonance lymphography of sentinel lymph nodes in patients with breast cancer using superparamagnetic iron oxide: a feasibility study. Breast Cancer 2014;21:394-401.

87. Cox K, Sever A, Jones S, et al. Validation of a technique using microbubbles and contrast enhanced ultrasound (CEUS) to biopsy sentinel lymph nodes (SLN) in preoperative breast cancer patients with a normal grey-scale axillary ultrasound. Eur J Surg Oncol 2013;39:760.

88. Sever A, Jones S, Cox K, et al. Preoperative localization of sentinel lymph nodes using intradermal microbubbles and contrast-enhanced ultrasonography in patients with breast cancer. Br J Surg 2009;96:1295-9.

89. Sever AR, Mills P, Jones SE, et al. Preoperative sentinel node identification with ultrasound using microbubbles in patients with breast cancer. AJR Am J Roentgenol 2011;196:251-6.

90. Gkegkes ID, Iavazzo C. Contrast enhanced ultrasound (CEU) using microbubbles for sentinel lymph node biopsy in breast cancer: a systematic review. Acta Chir Belg 2015;115:212.

91. Xie F, Zhang D, Cheng L, et al. Intradermal microbubbles and contrast-enhanced ultrasound (CEUS) is a feasible approach for sentinel lymph node identification in earlystage breast cancer. World J Surg Oncol 2015;13:319.

92. Matsuzawa F, Omoto K, Einama T. Accurate evaluation of axillary sentinel lymph node metastasis using contrastenhanced ultrasonography with Sonazoid in breast cancer: a preliminary clinical trial. Springerplus 2015;4:509.

93. Dellaportas D, Koureas A, Contis J. Contrast-enhanced color doppler ultrasonography for preoperative evaluation of sentinel lymph node in breast cancer patients. Breast Care (Basel) 2015;10:331-5.

94. Nielsen Moody A, Bull J, Culpan AM. Preoperative sentinel lymph node identification, biopsy and localisation using contrast enhanced ultrasound (CEUS) in patients with breast cancer: a systematic review and meta-analysis. Clin Radiol 2017;72:959-71.

95. Fang J, Ma L, Zhang YH, et al. Endoscopic sentinel lymph node biopsy and endoscopic axillary lymphadenectomy without liposuction in patients with early stage breast cancer. Surg Oncol 2017;26:338-44.

Cite this article as: Ferrucci M, Franceschini G, Douek M. New techniques for sentinel node biopsy in breast cancer. Transl Cancer Res 2018;7(Suppl 3):S405-S417. doi: 10.21037/ tcr.2018.02.07 